\title{
Postural Instability and Cognitive Dysfunction in Early Parkinson's Disease
}

\author{
Jong Moon Lee, Seong-Beom Koh, Sung Won Chae, Woo-Keun Seo, \\ Do Young Kwon, Ji Hyun Kim, Kyungmi Oh, Jong Sam Baik, Kun Woo Park
}

\begin{abstract}
Background: Postural instability is one of the most disabling features of Parkinson's disease, usually occurring in late and advanced stages. The aim of this study was to investigate the postural performance of early-stage de novo Parkinson's disease patients with no clinical postural instability using computerized dynamic posturography. We sought to understand the relationship between postural sway and disease severity and the relationship between postural instability quantitatively measured by computerized dynamic posturography and cognitive impairment in early-stage Parkinson's disease patients. Method: Thirty-one subjects with Parkinson's disease and 20 healthy controls were assessed by the computerized dynamic posturography protocol using the sensory organization test and the motor control test. A neuropsychological assessment was also administered. Results: The mean equilibrium score for sensory organization test and the vestibular input ratio were significantly correlated with Hoehn-Yahr stage. No associations between motor latency for any motor control test condition and Hoehn-Yahr stage were found. The equilibrium score for sensory organization test correlated with the mini-mental status examination scores. There was a significant correlation between motor latency for large backward translation and mini-mental status examination scores. There were significant correlations between visual perception/ construction/ memory of the neuropsychological battery test and the equilibrium score for sensory organization test and between verbal word learning test, controlled word association test and motor latency for large backward translation. Conclusion: These findings showed the postural instability present in early-stage (Hoehn-Yahr stage 2-2.5) Parkinson's disease. We also found a close relationship between postural instability and cognitive function in Parkinson's disease patients.
\end{abstract}

RÉSUMÉ: Instabilité posturale et dysfonction cognitive au stade précoce de la maladie de Parkinson. Contexte : L'instabilité posturale est l'une des caractéristique les plus invalidantes de la maladie de Parkinson (MP). Elle survient habituellement tard dans l'évolution de la maladie. Le but de cette étude était d'examiner la performance posturale de patients atteints de novo de la MP au moyen de la posturographie dynamique informatisée, au stade précoce de la maladie alors qu'il n'existe pas d'instabilité posturale détectable en clinique. Nous voulions examiner la relation entre les oscillations posturales et la sévérité de la maladie et entre l'instabilité posturale mesurée quantitativement par posturographie dynamique informatisée et le déficit cognitif chez des patients au stade précoce de la MP. Méthode : Trente et un patients atteints de la MP et 20 sujets témoins en bonne santé ont été évalués au moyen d'un protocole de posturographie dynamique informatisée qui comportait un test d'organisation sensorielle et un test du contrôle moteur de l'équilibration. Une évaluation neuropsychologique a également été effectuée. Résultats : Le score moyen d'équilibre au test d'organisation sensorielle et le ratio de contribution vestibulaire étaient corrélés au stade de la maladie de Hoehn-Yahr. Nous n'avons pas observé d'association entre la latence motrice pour toute pathologie détectée au test du contrôle moteur de l'équilibration et le stade de Hoehn-Yahr. Le score d'équilibre pour le test d'organisation sensorielle était corrélé au score du Mini Mental State Examination (MMSE). Une corrélation significative a été observée entre la latence motrice lors de la translation arrière de la plate-forme et les scores du MMSE. Il existait des corrélations significatives entre la perception visuelle, la construction, la mémoire évaluées par la batterie de tests neuropsychologiques et le score d'équilibre au test d'organisation sensorielle, et entre le test d'apprentissage audio-verbal, le test d'association de mots et la latence motrice à la translation arrière de la plate-forme. Conclusion : Ces observations démontrent la présence d'instabilité posturale aux stades précoces de la MP (Hoehn-Yahr stade 2-2,5). Nous avons également observé une relation étroite entre l'instabilité posturale et la fonction cognitive chez des patients atteints de la MP.

Can J Neurol Sci. 2012; 39: 473-482

Postural instability is one of the most disabling features of Parkinson's disease (PD), usually occurring in the late and advanced stages of the disease. Stage III of the Hoehn-Yahr (HY) scale is defined by postural instability. ${ }^{1}$ The pathophysiology of postural instability in PD is still unknown and is most likely complex due to the involvement of many different neural structures. Currently, it is broadly accepted that disorders in many afferent and efferent postural systems, which normally contribute to balance control, can influence the complex pathophysiology underlying postural instability in PD. ${ }^{2,3}$ Examples include inadequately organized automatic postural reactions, poor anticipatory postural responses, difficulty in initiating compensatory steps, inappropriately directed protective arm movements, and defective somatosensory integration of afferent sensory information. . $^{2,5}$

From the Departments of Neurology (JML, SBK, WKS, DYK, JHK, KO, KWP), Otolaryngology-Head and Neck Surgery and Korea University College of Medicine (SWC); Department of Neurology (JSB), Sanggye Paik Hospital, Inje University College of Medicine, Seoul, Korea.

Received September 8, 2011. Final Revisions Submitted January 10, 2012. Correspondence to: Seong-Beom Koh, Department of Neurology, Korea University College of Medicine Guro Hospital, \#80 Guro-Dong, Guro-Ku, Seoul, Korea 152-703. E-mail : parkinson@korea.ac.kr 
The evaluation of postural instability is not standardized. The retropulsion test is commonly used to clinically evaluate postural control in PD patients. However, its value is limited due to a lack of normative criteria and subjective interpretation of the outcome..$^{6-8}$ Therefore, quantitative measurements should be used to perform clinical evaluations and to investigate the risk factors associated with postural instability. Computerized dynamic posturography (CDP) allows two categories of tests including the sensory organization test (SOT) and motor control test (MCT). The SOT assesses subject ability to use visual, vestibular or somatosensory information to maintain an upright stance under different sensory conditions. The MCT assesses motor reflexes triggered by abrupt platform motion by positioning the patient on a platform surrounded by a background..$^{9,10}$ Previous quantitative studies of postural instability of early-stage PD patients have yielded inconsistent results. Some demonstrated that early-stage PD subjects have normal postural control in static and dynamic conditions, ${ }^{11-14}$ while others suggest that early-stage PD patients have infraclinical postural instability. ${ }^{15,16}$ These conflicting results stemmed from differences in disease severity, levodopa (L-dopa) treatment conditions, and method of postural instability (PI) quantification. The effects of L-dopa on tremor, rigidity, and bradykinesia were relatively consistent in these studies. In contrast, midline or axial symptoms associated with bulbar function, gait, balance and speech in advanced PD patients, which are poorly responsive to L-dopa medication, were due to non-dopaminergic deficits. ${ }^{17}$ It was previously demonstrated that L-dopa treatment could increase postural sway abnormalities. ${ }^{18,19}$

Two major clinical subtypes of PD are the postural instability/gait disturbance (PIGD)-dominant type and the tremor-dominant type. ${ }^{20}$ Several studies investigating the relationship between PIGD and dementia have shown that PIGD is more prevalent in demented than in non-demented PD patients, and that impairment of speech and balance at baseline predicted the incidence of dementia in PD patients. ${ }^{21-27}$ These studies administered clinical assessments including PD subtype defined by calculating Unified Parkinson's Disease Rating Scale (UPDRS) subscores and initial motor symptoms for the PDsubject grouping.

The aim of this study was to investigate CDP performance in the early-stage PD patients with no clinical postural instability or history of L-dopa/ anti-PD medication compared to age- and sexmatched controls. We sought to understand the relationship between postural sway and disease severity, and the relationship between postural instability quantitatively measured by CDP and cognitive impairment in early-stage PD patients.

\section{SubJects ANd Methods}

\section{Subjects}

Thirty-one subjects consecutively diagnosed with PD over a span of ten months in the Department of Neurology, Korea University Guro Hospital, were enrolled in the study. All patients met the United Kingdom Parkinson's Disease Society Brain Bank criteria for PD diagnosis. All patients were in early-stage $\mathrm{PD}$ (H-Y stage 1 to 2.5 ) with no history of L-dopa/anti-PD medication. Patients were screened for clinical signs of musculoskeletal and otoneurological problems, and peripheral sensory loss by a comprehensive history and physical examination. Polyneuropathy was excluded by clinical neurological assessments, including deep-tendon reflex and sensory testing. The PD group consisted of 16 women and 15 men, mean age $68.10 \pm 7.28$ years, and were divided into two groups on the basis of their modified H-Y score (group I: H-Y stage 1 and group II: H-Y stage 2 and 2.5). ${ }^{20}$ PD subjects were assessed using the SOT and the MCT of dynamic posturography (Neurocom, Clackamas, OR, USA). Clinical measures, such as the modified $\mathrm{H}-\mathrm{Y}$ stages and UPDRS III, were assessed immediately before CDP testing. A neuropsychological assessment was also administered to all patients.

Twenty control subjects were chosen to match the PD group with respect to age and gender. The age- and sex-matched control group consisted of ten women and ten men, mean age $66.60 \pm 7.80$. All control subjects were screened for neurological, vestibular, and peripheral sensory symptoms by patient history and physical examination, and all gave informed consent. The Korea University Institutional Review Board approved the study protocol.

\section{METHODS \\ Computerized dynamic posturography (CDP)}

Computerized dynamic posturography is a quantitative method for assessing balance under a variety of conditions that simulate conditions encountered in daily life. ${ }^{28}$ The NeuroCom Smart Equitest system was used to measure postural response to dynamic perturbations during SOT and MCT testing. The equipment is composed of a steel frame incorporating a dualforce plate system capable of translating in both the forwardbackward direction, causing rotation about the ankle joint in the sagittal plane when the support surface is sway referenced. A patterned screen that occupied the subject's field of view surrounded the subject while on the platform. The platform was equipped with force transducers that allowed measurement of the changes in subject weight distribution. The platform, equipped with force transducers, measures vertical force that along with the height of the patient, are used to calculate the center of gravity angle. The change in the center of gravity angle is measured in real time about the ankle joint.

Each subject's height was measured while he or she stood barefoot on the movable platform wearing a safety harness. Subjects were instructed to stand upright, face the visual surround, and maintain their balance throughout testing. The normal anterior to posterior range of sway was typically $12.5^{\circ}$ $\left(8.25^{\circ}\right.$ anterior, $4.25^{\circ}$ posterior) without the subject experiencing a loss of balance. ${ }^{29}$

Subject data were entered (age, height) into the NeuroCom software such that current results could be compared with the software age-matched normative data, and dynamic perturbations were scaled according to the subject's height.

\section{Sensory organization test $(\text { SOT })^{30,31}$}

The SOT objectively identifies problems with postural control by assessing the patients's ability to make effective use of visual, vestibular and proprioceptive information. Each subject's postural sway was measured during triplicate 20second trials after the following six sensory conditions: 
1. SOT1: Eyes open with fixed surface and visual surround.

2. SOT2: Eyes closed with a fixed surface.

3. SOT3: Eyes open with fixed surface and sway-referenced visual surround.

4. SOT4: Eyes open with sway-referenced surface and fixed visual surround.

5. SOT5: Eyes closed with a sway-referenced surface.

6. SOT6: Eyes open with sway-referenced surface and visual surround.

Equilibrium scores (0 100\%) during each trial and condition were measured and calculated by comparing the angular difference between the subject's measured maximum anterior to posterior center of gravity displacement to the theoretical sway stability limit of $12.5^{\circ}$. Loss of balance (score 0 ) occurred when a subject's sway reached and exceeded their stability limits and the subject took a step or required support to regain balance.

Subject sensory analysis shows the relative contribution of different sensorial inputs and is calculated by the equilibrium score obtained in the different test conditions. For example, visual input ratio reflects subject's ability to use visual input to maintain balance and visual preference ratio implies the degree to which a subject relies on visual information to maintain balance, even when the information is incorrect.

1. Somatosensory input ratio $=\mathrm{SOT} 2 / \mathrm{SOT} 1$

2. Visual input ratio $=\mathrm{SOT} 4 / \mathrm{SOT} 1$

3. Vestibular input ratio $=$ SOT5 $/$ SOT 1

4. Visual preference ratio $=(\mathrm{SOT} 3+\mathrm{SOT} 6) /(\mathrm{SOT} 2+\mathrm{SOT} 5)$

\section{Motor control test $(\mathrm{MCT})^{30,31}$}

For the MCT, the subjects' automatic postural reactions were measured in response to support surface translations. The surround remained stationary, and the subjects kept their eyes open throughout the test. The six conditions for MCT testing were both forward and backward platform translations to induce small $\left(2.8^{\circ} / \mathrm{s}\right)$, medium $\left(6.0^{\circ} / \mathrm{s}\right)$, and large $\left(8.0^{\circ} / \mathrm{s}\right)$ sway of the center of gravity, which were then measured at the onset of balance correction response. Responses were referred by long reflex arcs that affected both the tactile and muscular-tendinous receptor of the lower limbs, the sensitive and motor peripheral nerves, and the ascending and descending medullary pathways.

Latency scores from the MCT were determined by the differentiation of the force plate data from each foot. Postural response latency was the elapsed time between the onset of support surface translation until the subject actively resisted the induced sway.

\section{Neuropsychological assessments}

The general cognitive status of each subject was evaluated by means of the Korean versions of the mini-mental status examination (K-MMSE) and the clinical dementia rating (CDR) scale. Detailed cognition was evaluated using the Seoul Neuropsychological Screening Battery, ${ }^{32}$ which consists of an attention test (forward digit span, backward digit span, letter cancellation), a language and related function test (spontaneous speech, comprehension, repetition, naming measured by the Korean version of the Boston Naming test, reading, writing, finger naming, right-left orientation, body part identification, calculation, and praxis), a visuospatial function test (drawing interlocking pentagon and the Rey complex figure test), a verbal memory test (three word registration, three word recall, and the Seoul Verbal Learning Test which included immediate recall, delayed recall, recognition), a non-verbal memory test (immediate recall, delayed recall, and recognition of a Rey complex figure) and a frontal executive function test (motor impersistence, contrasting program, go-no-go test, fist-edgepalm, alternating hand movement, alternating square and triangle test, Luria loop, Controlled Oral Word Association Test: animal, supermarket and letter, Korean-color Word Stroop Test: word reading, color reading). Neuropsychological data were treated as continuous variables in an attempt to avoid biasing the results with categorization based on artificial cut-offs.

\section{Statistical analysis}

The data were expressed as means \pm standard deviation (SD) or $\mathrm{n}(\%)$. A $\mathrm{x}^{2}$ analysis was used for categorical variables. A Student's t-test was used to detect differences in the mean equilibrium scores (ES) in each SOT condition, and motor latency in each MCT condition between the control and earlystage PD groups. Kolmogorov-Smirnov test was used to detect the distribution of data. Because the data were not normally distributed, a Mann-Whitney rank sum test was used to determine significance between the ES for the control and the early-stage PD group. One-way analysis of variance (ANOVA) was used to identify significant differences in general distributions between the three groups (group I: H-Y stage 1, group II: H-Y stage $2 \& 2.5$, and control) and post hoc Tukey's $\mathrm{B}$ tests were used to explore precisely where the significant differences occurred. Kruskal-Wallis test was used to identify significant differences in CDP data between the three groups (group I: H-Y stage 1, group II: H-Y stage $2 \& 2.5$, and control). To test whether the mean ES in each SOT condition, and motor latency in each MCT condition were associated with H-Y stage, multilevel proportional odds ordinal regression modeling was conducted. Multivariate ordinal regression analyses were performed to control for the age and height covariates. A Spearman rank correlation was used to assess the relationship between the MMSE score and the ES in each SOT condition, between the posture score from the UPDRS motor scale and motor latency in each MCT condition and between the MMSE score and motor latency in each MCT condition. A P value of $\leq$ 0.05 was used as the level of significance. All analyses were performed using SPSS 12.0 for Windows.

\section{RESULTS}

Parkinson's disease patient demographics are summarized in Table 1. There were no differences in age, sex ratio, or height between PD patients and controls. The average H-Y stage of early-stage PD patients was $1.8 \pm 0.50$. When PD patients were divided into two groups on the basis of modified $\mathrm{H}-\mathrm{Y}$ scores (group I: H-Y stage 1, group II: H-Y stage $2 \& 2.5$ ), there were no differences in age, sex ratio, or height among group I, group II, and the controls (Table 1). The mean score of MMSE of PD patients was $23.6 \pm 3.6$ (range, 17-29).

Under all SOT conditions, comparison between early-stage PD subjects and the control group did not show any significant differences in mean ES, and the sensory analysis ratio results did not show any significant differences between groups (Table 2). No significant differences in motor latency under any MCT 
Table 1: Demographic and clinical characteristics of subgroups of PD patients according to $\mathrm{H}$ Y stage and controls

\begin{tabular}{|c|c|c|c|c|}
\hline & Group I & Group II & Controls & $P$-value \\
\hline & H-Y $1(n=10)$ & H-Y 2 and $2.5 \quad(n=21)$ & $(n=20)$ & \\
\hline Age $(\text { years })^{\mathrm{a}}$ & $67.26 \pm 6.49$ & $68.51 \pm 7.75$ & $66.60 \pm 7.80$ & 0.718 \\
\hline Sex $(\text { Male/Female })^{b}$ & $5 / 5$ & $10 / 11$ & $10 / 10$ & 0.986 \\
\hline $\operatorname{Height}(\mathrm{cm})^{\mathrm{a}}$ & $157.80 \pm 8.05$ & $159.52 \pm 9.34$ & $159.50 \pm 9.18$ & 0.866 \\
\hline Disease duration(months) ${ }^{c}$ & $15.10 \pm 10.48$ & $14.57 \pm 16.25$ & - & 0.926 \\
\hline Age at onset(years) ${ }^{c}$ & $64.90 \pm 6.90$ & $67.24 \pm 8.63$ & - & 0.460 \\
\hline Education $\left(\right.$ years) ${ }^{\mathrm{c}}$ & $6.10 \pm 4.80$ & $6.93 \pm 4.50$ & - & 0.642 \\
\hline UPDRS motor scores & $8.20 \pm 2.35$ & $19.88 \pm 5.90$ & & $<0.001$ \\
\hline
\end{tabular}

PD, Parkinson's disease; H-Y stage, Hoehn-Yahr stage. Data are expressed as mean \pm standard deviation. ${ }^{a}$

One-way ANOVA test. ${ }^{\mathrm{b}} \mathrm{x} 2$ test. ${ }^{\mathrm{c}}$ Student's t-test.

condition were detected between the early-stage PD subjects and the control group (Table 2). When the early-stage PD subjects were divided into groups I and II (based on H-Y scale) and compared with controls, no significant differences in the mean ES and motor latency among the three subgroups was found (Table 2). In SOT5 and vestibular input ratio, group II showed a trend that was lower than the controls and group I, but the overall differences were not significant (SOT5, $\mathrm{P}=0.082$; vestibular input ratio, $\mathrm{P}=0.061$ ) (Table 2 ).

Results of the multilevel proportional odds ordinal regression analyses are shown in Table 3. Results showed the mean ES in SOT5 and vestibular input ratios had significant negative associations with UPDRS motor scores. Thus, postural sway increased with increasing disease severity in the SOT5 condition (Table 3, supplementary Figure 1). There was no association between latency of any MCT condition and H-Y stage (supplementary Table 1). Of all MCT conditions, there was a significant correlation between the motor latency score for large backward translation and posture item of UPDRS motor scale $(\gamma=0.474, \mathrm{P}<0.05)$

There were significant correlations between the ES for SOT4 to SOT6 condition and the MMSE scores (supplementary Figure 2 ). The correlation was only seen in sway-referenced floor conditions (SOT4, $r=0.404$; SOT5, $\gamma=0.383$; SOT6, $\gamma=0.446$, $\mathrm{P}<0.05)$. Thus, postural sway increased with more impaired general cognition under dynamic conditions. These differences translated into a significant correlation between visual input or vestibular input and MMSE scores (Figure 1). There was a significant correlation between the motor latency for large backward translation and MMSE scores (Figure 2, $\gamma=-0.452, \mathrm{P}$ $=0.011)$. Thus, motor latency for large backward translation increased with more impaired general cognition.

There were significant correlations between several domains of the detailed neuropsychological battery test such as attention, naming, visual memory and verbal memory and the ES in the

Table 2: Sensory organization test (SOT) and motor control test (MCT) data in PD patients according to $\mathrm{H}-\mathrm{Y}$ stage and controls

\begin{tabular}{|c|c|c|c|c|c|c|c|}
\hline \multirow{2}{*}{ SOT condition $(\%)^{\mathrm{a}}$} & \multicolumn{2}{|c|}{$\begin{array}{c}\text { Group I } \\
\text { H-Y } 1(n=10)\end{array}$} & \multicolumn{2}{|c|}{ Group II } & \multicolumn{2}{|c|}{$\begin{array}{c}\text { Controls } \\
(n=20)\end{array}$} & $P$-value \\
\hline & \multirow{2}{*}{\multicolumn{2}{|c|}{$91.48 \pm 1.77$}} & \multirow{2}{*}{\multicolumn{2}{|c|}{$93.21 \pm 2.78$}} & & & \\
\hline SOT 1 & & & & & \multicolumn{2}{|c|}{$93.29 \pm 3.02$} & 0.196 \\
\hline SOT 2 & \multicolumn{2}{|c|}{$88.53 \pm 2.38$} & \multicolumn{2}{|c|}{$88.85 \pm 5.46$} & \multicolumn{2}{|c|}{$89.82 \pm 5.74$} & 0.758 \\
\hline SOT 3 & \multicolumn{2}{|c|}{$85.88 \pm 7.57$} & \multicolumn{2}{|c|}{$87.85 \pm 5.89$} & \multicolumn{2}{|c|}{$88.38 \pm 6.92$} & 0.621 \\
\hline SOT 4 & \multirow{2}{*}{\multicolumn{2}{|c|}{$\begin{array}{l}73.53 \pm 7.23 \\
58.08+8.01\end{array}$}} & \multicolumn{2}{|c|}{$73.36 \pm 9.78$} & \multicolumn{2}{|c|}{$76.03 \pm 7.30$} & 0.559 \\
\hline SOT 5 & & & \multirow{2}{*}{\multicolumn{2}{|c|}{$\begin{array}{l}47.26 \pm 23.74 \\
43.95 \pm 30.05\end{array}$}} & \multicolumn{2}{|c|}{$58.54 \pm 10.52$} & 0.082 \\
\hline SOT 6 & \multicolumn{2}{|c|}{$53.78 \pm 14.23$} & & & \multicolumn{2}{|c|}{$54.60 \pm 14.95$} & 0.275 \\
\hline \multicolumn{8}{|l|}{ Sensory analysis $(\%)^{\mathrm{a}}$} \\
\hline Somatosensory input & \multicolumn{2}{|c|}{$96.79 \pm 2.73$} & \multicolumn{2}{|c|}{$95.28 \pm 4.30$} & \multicolumn{2}{|c|}{$96.22 \pm 4.33$} & 0.582 \\
\hline Visual input & \multicolumn{2}{|c|}{$80.40 \pm 7.89$} & \multicolumn{2}{|c|}{$78.63 \pm 9.73$} & \multicolumn{2}{|c|}{$81.51 \pm 7.47$} & 0.559 \\
\hline Vestibular input & \multicolumn{2}{|c|}{$63.52 \pm 8.92$} & \multicolumn{2}{|c|}{$50.43 \pm 25.26$} & \multicolumn{2}{|c|}{$62.83 \pm 11.41$} & 0.061 \\
\hline Visual preference & \multicolumn{2}{|c|}{$95.03 \pm 8.67$} & \multicolumn{2}{|c|}{$96.93 \pm 14.92$} & \multicolumn{2}{|c|}{$96.52 \pm 11.73$} & 0.925 \\
\hline$M C T$ condition $(m s)^{a}$ & & & & & & & \\
\hline Small backward & 153.00 & 11.35 & 150.24 & 17.36 & 153.00 & 16.34 & 0.832 \\
\hline Forward & 165.00 & 13.12 & 155.00 & 13.87 & 153.75 & 12.97 & 0.087 \\
\hline Medium backward & 142.00 & 12.95 & 142.38 & 8.89 & 136.75 & 10.55 & 0.193 \\
\hline Forward & 140.00 & 12.69 & 133.36 & 12.38 & 140.50 & 9.72 & 0.112 \\
\hline Large backward & 138.00 & 8.23 & 132.86 & 19.21 & 131.75 & 8.47 & 0.502 \\
\hline Forward & 139.00 & 14.49 & 132.98 & 12.84 & 133.75 & 6.46 & 0.357 \\
\hline
\end{tabular}

SOT, Sensory organization test; MCT, Motor control test; PD, Parkinson's disease; H-Y stage, Hoehn-Yahr stage. Data are expressed as mean \pm standard deviation. ${ }^{a}$ Kruskal-Wallis test 


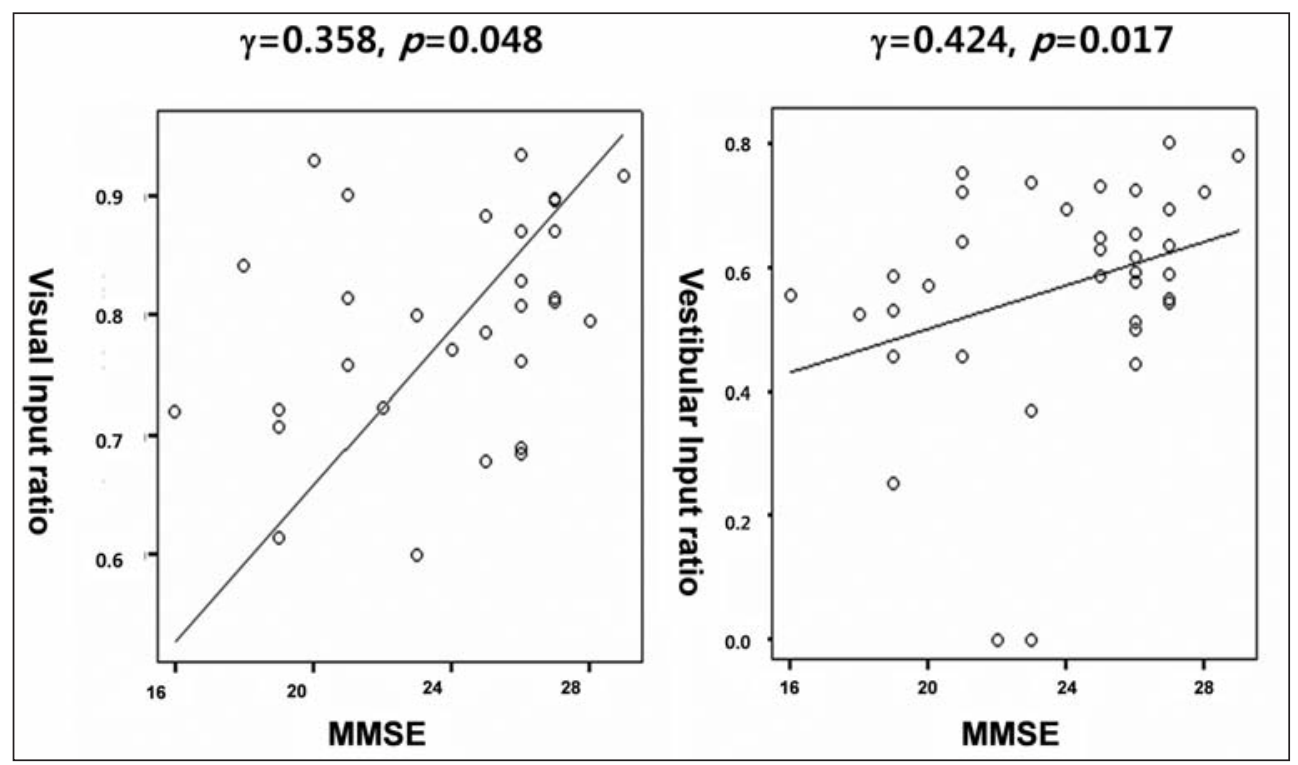

Figure 1: The correlation between MMSE score and Sensory Analysis ratio(visual input and vestibular input). MMSE, Mini-mental state examination.

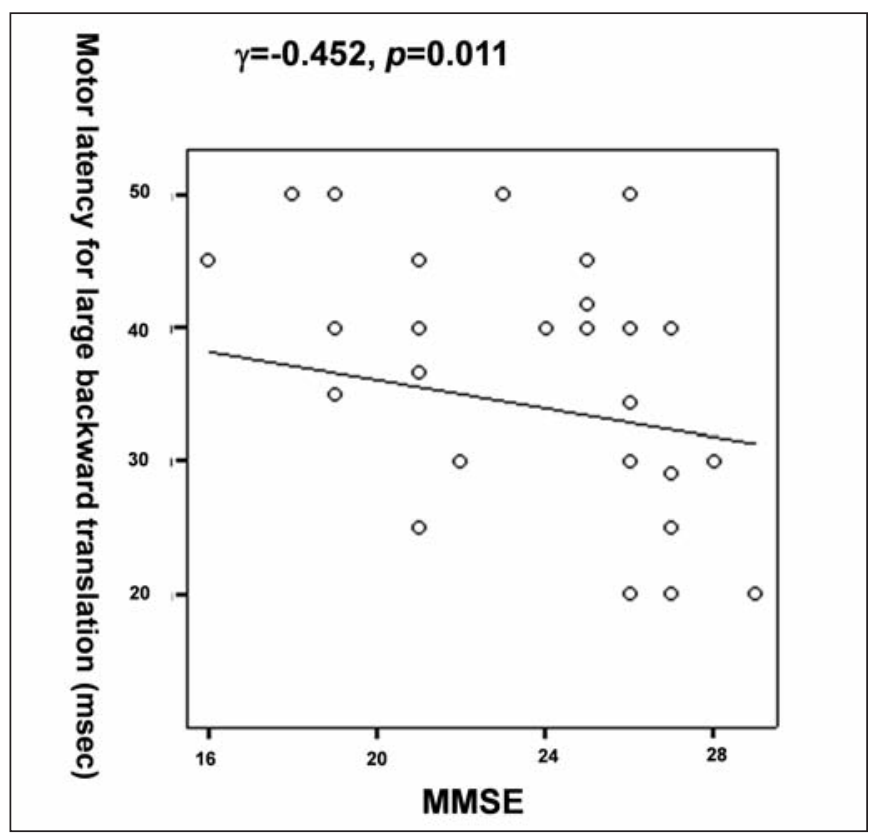

Figure 2: The correlation between MMSE Score and Motor latency for large backward translation. MMSE, Mini-mental state examination.

SOT2 to SOT6 condition, with sensory analysis ratios such as somatosensory, visual, and vestibular input or motor latency for large backward translation (Supplementary Table 2,3). Again, SOT5 and vestibular input were correlated with disease severity (H-Y stage) in early-stage PD patients. It was observed that the ES in SOT5 correlated with the following two test scores: Rey complex figure test (R-CFT) $(\gamma=0.416, \mathrm{P}=0.02)$ and immediate recall of R-CFT ( $\gamma=0.472, \mathrm{P}=0.007)$. The scores of vestibular input correlated with the following three test scores: R-CFT $(\gamma=$
0.444, $\mathrm{P}=0.012)$, immediate recall $(\gamma=0.513, \mathrm{P}=0.003)$ and delayed recall of R-CFT $(\gamma=0.436, \mathrm{P}=0.014)$.

\section{DiscusSION}

We sought to determine whether a significant difference of postural instability measured by a CDP method was present between two H-Y-distinguished groups of early-stage PD subjects and healthy controls, and how this postural instability correlated with cognitive performance. All patients were at the early stage of disease (Hoehn-Yahr stage 1 to 2.5) with no history of L-dopa medication.

\section{Normal postural control in early-stage PD subjects}

There is controversy in the literature as to whether postural instability measured by CDP in early-stage PD patients is different from healthy controls. ${ }^{11,12,15,33,34}$ In this study, we show that early-stage PD subjects had normal ES values under any SOT condition and normal motor latency under any MCT condition. Our patients had received no L-dopa medication; therefore we could exclude the effect or side effects of this treatment in our assessment. We found no significant differences in postural instability measured by CDP between early-stage PD subjects and healthy controls. This finding suggests that the measure of postural sway by CDP may be an objective method to observe PD progression, as patients are normal at early stages of the disease and become increasingly abnormal with disease progression. Frenklach, A et al reported.

\section{Relationships between ES for SOT5, or vestibular input and H- $Y$ stage in early stage $P D$}

The ES for SOT5 weakly correlated with H-Y stage, and the vestibular input ratio was more associated with increased $\mathrm{H}-\mathrm{Y}$ stage than SOT5 condition, similar to the Frenklach's study of untreated PD patients. (Supplementary Table 4) This association persisted after adjusting for significant covariates such as age 
Table 3: The relationship between the mean ES in each SOT condition, and sensory analysis ratio and UPDRS motor score in PD patients, and controls: a Multilevel Ordinal Regression Model

\begin{tabular}{|c|c|c|c|c|}
\hline & Estimate & Standard Error & Univariate $P$-value $^{\mathrm{a}}$ & Multivariate $P$-value ${ }^{\mathrm{b}}$ \\
\hline \multicolumn{5}{|l|}{ SOT condition(\%) } \\
\hline SOT 1 & -0.037 & 0.094 & 0.691 & 0.843 \\
\hline SOT 2 & -0.064 & 0.052 & 0.219 & 0.394 \\
\hline SOT 3 & -0.022 & 0.039 & 0.584 & 0.690 \\
\hline SOT 4 & -0.051 & 0.032 & 0.115 & 0.113 \\
\hline SOT 5 & -0.045 & 0.017 & $0.007 *$ & $0.008 *$ \\
\hline SOT 6 & -0.022 & 0.012 & 0.063 & 0.090 \\
\hline \multicolumn{5}{|l|}{ Sensory analysis ratio } \\
\hline Somatosensory input & -7.957 & 6.548 & 0.224 & 0.364 \\
\hline Visual input & -4.990 & 3.153 & 0.114 & 0.092 \\
\hline Vestibular input* & -4.258 & 1.552 & $0.006^{*}$ & $0.007^{*}$ \\
\hline Visual preference & 0.269 & 2.079 & 0.897 & 0.827 \\
\hline
\end{tabular}

SOT, Sensory organization test; PD, Parkinson's disease; UPDRS, Unified Parkinson's disease Rating Scale. Data are expressed as mean \pm standard deviation. ${ }^{a}$ Univariate ordinal regression analyses. ${ }^{\mathrm{b}}$ Multivariate ordinal regression analyses were performed to control for the covarirates such as age and height. ${ }^{*} \mathrm{P}<0.05$

and height, which probably played a role in its instability. Measurement of postural sway by CDP might be an objective method to observe PD progression in early stages of the disease. Because PD is a central nervous system disorder, this deficiency suggests a dysfunction in central processing rather than a peripheral lesion. The deficit in SOT5 or vestibular input ratio cannot be explained by peripheral or brainstem vestibular disease in this study, as none of the patients had clinical signs of vestibular disease. If peripheral vestibular sensory inputs are normal in PD, a possible explanation for the association of vestibular dysfunction could be deficits in central vestibular or sensorimotor integration. ${ }^{19,34}$ Certain studies have suggested that PD subjects are highly visually dependent..$^{35,36}$ The measurement of visual dependence can be achieved using SOT by comparing ES under conditions of visual absence with conditions where visual feedback is incongruent; however, in this study a specific visually-dependent group of the early-stage PD patients was not identified.

\section{Relationship between ES for SOT4, 5, 6, visual input ratio, or vestibular input ratio and MMSE in early stage $P D$}

The SOT4, 5, 6 pattern has been labeled 'surface dependence' or 'combined visual-vestibular deficit'. Patients with this pattern were unable to stand when somatosensation was distorted despite having the opportunity for normal visual and vestibular inputs. ${ }^{9}$ Under dynamic conditions, PD subjects are unable to use the proprioceptive feedback for orientation. Previous studies suggested that the expressed postural instability of PD patients under these conditions is attributed to a failure of higher-order sensory integration centers rather than an abnormality in primary vestibular feedback, as this is normal in PD. ${ }^{12,37}$ These results suggested that postural instability in dynamic conditions became worse with increasingly impaired cognition. Postural sway increased with an increase in impaired general cognition under dynamic conditions. These differences translated into a significant correlation between visual input or vestibular input and MMSE scores.

\section{Relationships between motor latency for large backward} translations and MMSE in early-stage PD

The MCT measured automatic postural responses to rapid unexpected movements of the force plate. This study showed that motor latency was not significantly different between PD subjects and controls, and was not correlated with disease severity; however, the scores of motor latency for large backward translation were negatively correlated with cognitive impairment, and positively correlated with posture item of motor UPDRS scale. It is thought that the correlation of motor latency with only backward translation and MMSE could be due to the stooped posture, one of the parkinsonian features. The nervous system, including high cortical function, normally makes a "best guess" about an anticipated postural perturbation and primes an appropriate postural response before the response occurs. Postural instability evaluated via the scores of motor latency for large backward transition might be an indicator of reduced postural stability related to cognitive decline in PD.

Relationships between each domain of the detailed neuropsychological battery test and the ES for each SOT condition: sensory analysis ratios and motor latencies for each MCT condition

There were several significant correlations between each domain of the detailed neuropsychological test and the ES for each SOT condition, sensory analysis ratios, or motor latency for each MCT condition. The SOT5 or vestibular input that correlated with disease severity in early-stage PD subjects correlated with R-CFT copy and recall of R-CFT. With these exceptions, the ES in several conditions or the ratios of sensory analysis correlated with several domains of the neuropsychological test. It is thought that postural instability could be related to visuospatial function, or visual memory. These findings might be affected by the influence of each characteristic of the conditions of CDP themselves. Therefore, generalized cognitive function might correlate with postural sway. 


\section{Study Limitation}

It must be acknowledged that there were several limitations to the current study. Although PD patients and controls did not differ in mean scores on the SOT and MLT, we found that a within-group PD analysis yielded significant correlations with $\mathrm{H}-\mathrm{Y}$ stage. This finding could explain not only cognitive dysfunction but also increased variance in the PD group with some patients having worse than normal and others better than normal performance. For example, it appeared from Table 2 that the SD for some tests (e.g., SOT $5 \& 6$ and the vestibular input ratio and preference measures) was much higher in the $\mathrm{H}-\mathrm{Y}$ stage 2-2.5 patient group compared to the HY stage I group and normal control. This implied that our findings in fact were largely driven by the patients in HY 2-2.5 patients rather than the real early PD patient (H-Y stage 1), and that the increased variance was caused by vestibulr over-compesation in some patients and/or decompensation in others.

\section{Conclusions}

We used CDP to quantify postural instability in early-stage PD patients. No significant difference between early-stage PD subjects and controls was identified; however, ES in SOT5 or the vestibular input ratio correlated with the severity of early-stage PD. We demonstrated that there was a negative correlation between the ES in SOT4 to SOT6 and the MMSE. SOT5 and vestibular input was correlated with visuospatial dysfunction and visual memory dysfunction. These findings suggested that CDP could not differentiate postural instability in early-stage PD, and infraclinical postural instability might exist in early-stage PD. This study showed the subclinical abnormalities in balance and equilibrium in the early-stage PD patients with no clinical postural instability. We thought that the subclinical balance abnormality might relate to cognitive dysfunctions.

\section{ACKNOWLEDGEMENTS}

This work was supported by the basic Science Research Program through the National Research Foundation of Korea funded by the Ministry of Education, Science and Technology (2010-0027994).

\section{REFERENCES}

1. Hoehn MM, Yahr MD. Parkinsonism: onset, progression and mortality. Neurology. 1967 May;17(5):427-42.

2. Boonstra TA, van der Kooij H, Munneke M, Bloem BR. Gait disorders and balance disturbances in Parkinson's disease: clinical update and pathophysiology. Curr Opin Neurol. 2008 Aug;21(4):461-71.

3. Grimbergen YA, Munneke M, Bloem BR. Falls in Parkinson's disease. Curr Opin Neurol. 2004 Aug;17(4):405-15.

4. Bloem BR. Postural instability in Parkinson's disease. Clin Neurol Neurosurg. 1992;94 Suppl:S41-5.

5. Benatru I, Vaugoyeau M, Azulay JP. Postural disorders in Parkinson's disease. Neurophysiol Clin. 2008 Dec;38(6):459-65.

6. Bloem BR, Grimbergen YA, Cramer M, Willemsen M, Zwinderman AH. Prospective assessment of falls in Parkinson's disease. J Neurol. 2001 Nov;248(11):950-8.

7. Munhoz RP, Li JY, Kurtinecz M, et al. Evaluation of the pull test technique in assessing postural instability in Parkinson's disease. Neurology. 2004 Jan 13;62(1):125-7.

8. Visser M, Marinus J, Bloem BR, Kisjes H, van den Berg BM, van Hilten JJ. Clinical tests for the evaluation of postural instability in patients with parkinson's disease. Arch Phys Med Rehabil. 2003 Nov;84(11):1669-74.
9. Furman JM. Posturography: uses and limitations. Baillieres Clin Neurol. 1994 Nov;3(3):501-13.

10. Artuso A, Garozzo A, Contucci AM, Frenguelli A, Di Girolamo S. Role of dynamic posturography (Equitest) in the identification of feigned balance disturbances. Acta Otorhinolaryngol Ital. $2004 \mathrm{Feb} ; 24(1): 8-12$.

11. Waterston JA, Hawken MB, Tanyeri S, Jantti P, Kennard C. Influence of sensory manipulation on postural control in Parkinson's disease. J Neurol Neurosurg Psychiatry. 1993 Dec; 56(12):1276-81.

12. Frenklach A, Louie S, Koop MM, Bronte-Stewart H. Excessive postural sway and the risk of falls at different stages of Parkinson's disease. Mov Disord. 2009 Feb 15;24(3):377-85.

13. Valkovic P, Krafczyk S, Botzel K. Postural reactions to soleus muscle vibration in Parkinson's disease: scaling deteriorates as disease progresses. Neurosci Lett. 2006 Jun 19;401(1-2):92-6.

14. Valkovic P, Abrahamova D, Hlavacka F, Benetin J. Static posturography and infraclinical postural instability in early-stage Parkinson's disease. Mov Disord. 2009 Aug 15;24(11):1713-4; author reply 4.

15. Chastan N, Debono B, Maltete D, Weber J. Discordance between measured postural instability and absence of clinical symptoms in Parkinson's disease patients in the early stages of the disease. Mov Disord. 2008 Feb 15;23(3):366-72.

16. Fioretti S, Guidi M, Ladislao L, Ghetti G. Analysis and reliability of posturographic parameters in Parkinson patients at an early stage. Conf Proc IEEE Eng Med Biol Soc. 2004;1:651-4.

17. Lang AE, Obeso JA. Challenges in Parkinson's disease: restoration of the nigrostriatal dopamine system is not enough. Lancet Neurol. 2004 May;3(5):309-16.

18. Rocchi L, Chiari L, Horak FB. Effects of deep brain stimulation and levodopa on postural sway in Parkinson's disease. J Neurol Neurosurg Psychiatry. 2002 Sep;73(3):267-74.

19. Bronte-Stewart HM, Minn AY, Rodrigues K, Buckley EL, Nashner LM. Postural instability in idiopathic Parkinson's disease: the role of medication and unilateral pallidotomy. Brain. 2002 Sep; 125(Pt 9):2100-14.

20. Jankovic J, McDermott M, Carter J, et al. Variable expression of Parkinson's disease: a base-line analysis of the DATATOP cohort. The Parkinson Study Group. Neurology. 1990 Oct;40 (10):1529-34.

21. Alves G, Larsen JP, Emre M, Wentzel-Larsen T, Aarsland D. Changes in motor subtype and risk for incident dementia in Parkinson's disease. Mov Disord. 2006 Aug;21(8):1123-30.

22. Burn DJ, Rowan EN, Allan LM, Molloy S, O'Brien JT, McKeith IG. Motor subtype and cognitive decline in Parkinson's disease, Parkinson's disease with dementia, and dementia with Lewy bodies. J Neurol Neurosurg Psychiatry. 2006 May;77(5):585-9.

23. Gago MF, Garrett MC, Fonseca MR, et al. How do cognitive and axial motor signs correlate in Parkinson's disease? A 6-year prospective study. J Neurol. 2009 Oct;256(10):1655-62.

24. Levy G, Tang MX, Cote LJ, et al. Motor impairment in PD: relationship to incident dementia and age. Neurology. 2000 Aug 22:55(4):539-44.

25. Lyros E, Messinis L, Papathanasopoulos P. Does motor subtype influence neurocognitive performance in Parkinson's disease without dementia? Eur J Neurol. 2008 Mar;15(3):262-7.

26. Oh JY, Kim YS, Choi BH, Sohn EH, Lee AY. Relationship between clinical phenotypes and cognitive impairment in Parkinson's disease (PD). Arch Gerontol Geriatr. 2009 Nov-Dec;49(3):351-4.

27. Williams LN, Seignourel P, Crucian GP, et al. Laterality, region, and type of motor dysfunction correlate with cognitive impairment in Parkinson's disease. Mov Disord. 2007 Jan;22(1):141-5.

28. Nashner LM. Practical management of the dizzy patient. In: Goebel JA, editor. Computerized dynamic posturography. Philadelphia; 2001. p. 143-70.

29. Nashner LM, Shupert CL, Horak FB, Black FO. Organization of posture controls: an analysis of sensory and mechanical constraints. Prog Brain Res. 1989;80:411-8; discussion 395-7.

30. Hamid MA, Hughes GB, Kinney SE. Specificity and sensitivity of dynamic posturography. A retrospective analysis. Acta Otolaryngol Suppl. 1991;481:596-600. 
31. Horak FB, Nashner LM. Central programming of postural movements: adaptation to altered support-surface configurations. J Neurophysiol. 1986 Jun;55(6):1369-81.

32. Ahn HJ, Chin J, Park A, et al. Seoul Neuropsychological Screening Battery-dementia version (SNSB-D): a useful tool for assessing and monitoring cognitive impairments in dementia patients. J Korean Med Sci. Jul;25(7):1071-6.

33. Ondo W, Warrior D, Overby A, et al. Computerized posturography analysis of progressive supranuclear palsy: a case-control comparison with Parkinson's disease and healthy controls. Arch Neurol. 2000 Oct:57(10):1464-9.

34. Rossi M, Soto A, Santos S, Sesar A, Labella T. A prospective study of alterations in balance among patients with Parkinson's Disease. Protocol of the postural evaluation. Eur Neurol. 2009; 61(3):171-6.

35. Azulay JP, Mesure S, Amblard B, Blin O, Sangla I, Pouget J. Visual control of locomotion in Parkinson's disease. Brain. 1999 Jan; 122(Pt 1):111-20.

36. Isableu B, Ohlmann T, Cremieux J, Amblard B. Selection of spatial frame of reference and postural control variability. Exp Brain Res. 1997 May;114(3):584-9.

37. Pastor MA, Day BL, Marsden CD. Vestibular induced postural responses in Parkinson's disease. Brain. 1993 Oct;116(Pt 5): 1177-90.

\section{Supplementary Table 1: The relationship between Motor control test (MCT) results of latencies and $\mathrm{H}-\mathrm{Y}$ stage in PD patients, and controls: a Multilevel Ordinal Regression Model}

\begin{tabular}{cccc}
\hline & Estimate & Standard Error & Univariate $P$-value $^{\mathrm{a}}$ \\
\hline MCT condition & & & \\
Small backward & 0.009 & 0.007 & 0.230 \\
Forward & 0.007 & 0.019 & 0.701 \\
Medium backward & 0.043 & 0.026 & 0.095 \\
Forward & -0.037 & 0.023 & 0.108 \\
Large backward & 0.010 & 0.019 & 0.587 \\
Forward & -0.006 & 0.023 & 0.806 \\
\hline
\end{tabular}

MCT, Motor control test; H-Y stage, Hoehn-Yahr stage; PD, Parkinson's disease.

${ }^{\text {a }}$ Univariate ordinal regression analyses

Supplementary Table 2: The correlation between ES for each SOT condition and each domain of neuropsychological test

\begin{tabular}{|c|c|c|c|c|c|c|c|c|c|c|c|c|c|c|c|}
\hline$E S^{a}$ & & $\begin{array}{c}\text { Digit } \\
\text { span } \\
\text { forward }\end{array}$ & $\begin{array}{l}\text { Digit span } \\
\text { backward }\end{array}$ & $\begin{array}{l}\text { Naming } \\
\text { (K-BNT) }\end{array}$ & $\begin{array}{l}\text { R-CFT } \\
\text { copy }\end{array}$ & $\begin{array}{c}\text { SVLT } \\
\text { free } \\
\text { recall }\end{array}$ & $\begin{array}{c}\text { SVLT } \\
\text { delayed recall }\end{array}$ & $\begin{array}{l}\text { SVLT } \\
\text { recogni- } \\
\text { tion }\end{array}$ & $\begin{array}{c}\mathrm{R} \text {-CFT } \\
\text { immediate } \\
\text { recall }\end{array}$ & $\begin{array}{c}\text { R-CFT } \\
\text { delayed } \\
\text { recall }\end{array}$ & $\begin{array}{l}\text { R-CFT } \\
\text { recogniti } \\
\text { on }\end{array}$ & $\begin{array}{l}\text { COWAT } \\
\text { Category } \\
\text { fluency }\end{array}$ & $\begin{array}{c}\text { COWAT } \\
\text { Letter } \\
\text { fluency }\end{array}$ & $\begin{array}{c}\text { Stroop } \\
\text { word } \\
\text { reading }\end{array}$ & $\begin{array}{l}\text { Stroop } \\
\text { color } \\
\text { reading }\end{array}$ \\
\hline \multirow[t]{2}{*}{ SOT1 } & $\mathrm{CC}$ & 0.101 & 0.296 & -0.019 & -0.056 & 0.294 & 0.066 & 0.056 & -0.131 & -0.090 & -0.184 & 0.214 & 0.013 & -0.058 & 0.119 \\
\hline & $P$-value & 0.589 & 0.106 & 0.919 & 0.764 & 0.109 & 0.724 & 0.765 & 0.482 & 0.630 & 0.323 & 0.248 & 0.945 & 0.755 & 0.523 \\
\hline \multirow[t]{2}{*}{ SOT2 } & $\mathrm{CC}$ & 0.174 & $0.552^{* * *}$ & 0.175 & $0.391^{*}$ & $0.447^{*}$ & 0.156 & 0.220 & 0.244 & 0.236 & -0.044 & 0.332 & 0.172 & 0.137 & 0.296 \\
\hline & $P$-value & 0.348 & 0.001 & 0.345 & 0.029 & 0.012 & 0.401 & 0.235 & 0.186 & 0.201 & 0.813 & 0.068 & 0.354 & 0.461 & 0.106 \\
\hline \multirow[t]{2}{*}{ SOT3 } & $\mathrm{CC}$ & 0.147 & 0.317 & 0.071 & 0.040 & $0.414 *$ & 0.102 & 0.025 & 0.028 & -0.023 & -0.184 & $0.429^{*}$ & 0.132 & 0.008 & 0.207 \\
\hline & $P$-value & 0.429 & 0.082 & 0.703 & 0.833 & 0.020 & 0.585 & 0.892 & 0.883 & 0.902 & 0.322 & 0.016 & 0.477 & 0.967 & 0.263 \\
\hline \multirow[t]{2}{*}{ SOT4 } & $\mathrm{CC}$ & 0.242 & 0.304 & $0.471^{* *}$ & 0.260 & 0.308 & 0.128 & 0.054 & $0.402 *$ & $0.370^{*}$ & 0.020 & 0.319 & $0.495^{* * *}$ & 0.185 & 0.165 \\
\hline & $P$-value & 0.190 & 0.096 & 0.007 & 0.158 & 0.092 & 0.494 & 0.772 & 0.025 & 0.040 & 0.914 & 0.080 & 0.005 & 0.320 & 0.375 \\
\hline \multirow[t]{2}{*}{ SOT5 } & $\mathrm{CC}$ & -0.146 & 0.117 & 0.185 & $0.416^{*}$ & 0.309 & 0.280 & 0.259 & $0.472 * *$ & $0.390^{*}$ & 0.036 & 0.006 & 0.154 & 0.198 & 0.103 \\
\hline & $P$-value & 0.433 & 0.532 & 0.319 & 0.020 & 0.091 & 0.127 & 0.159 & 0.007 & 0.030 & 0.847 & 0.974 & 0.408 & 0.285 & 0.580 \\
\hline \multirow[t]{2}{*}{ SOT6 } & $\mathrm{CC}$ & 0.011 & 0.084 & 0.092 & $0.364 *$ & 0.302 & 0.161 & 0.164 & $0.457^{* * *}$ & $0.462 * *$ & -0.256 & 0.275 & 0.146 & -0.012 & 0.097 \\
\hline & $P$-value & 0.951 & 0.654 & 0.624 & 0.044 & 0.098 & 0.386 & 0.378 & 0.010 & 0.009 & 0.164 & 0.134 & 0.433 & 0.950 & 0.603 \\
\hline \multicolumn{16}{|l|}{$S A$} \\
\hline \multirow[t]{2}{*}{ somato } & $\mathrm{CC}$ & 0.126 & $0.447^{*}$ & 0.141 & $0.462^{* * *}$ & $0.419 *$ & 0.250 & 0.306 & 0.340 & 0.312 & -0.039 & 0.334 & 0.190 & 0.134 & 0.302 \\
\hline & $P$-value & 0.499 & 0.012 & 0.450 & 0.009 & 0.019 & 0.174 & 0.095 & 0.061 & 0.088 & 0.836 & 0.066 & 0.305 & 0.471 & 0.099 \\
\hline \multirow[t]{2}{*}{ visual } & $\mathrm{CC}$ & 0.202 & 0.288 & $0.462 * *$ & 0.259 & 0.254 & 0.128 & 0.055 & $0.435^{*}$ & $0.392^{*}$ & 0.050 & 0.294 & $0.491^{* * *}$ & 0.203 & 0.142 \\
\hline & $P$-value & 0.277 & 0.116 & 0.009 & 0.160 & 0.168 & 0.494 & 0.767 & 0.014 & 0.029 & 0.791 & 0.109 & 0.005 & 0.273 & 0.447 \\
\hline \multirow[t]{2}{*}{ vestibular } & $\mathrm{CC}$ & -0.140 & 0.093 & 0.182 & $0.444 *$ & 0.304 & 0.281 & 0.261 & $0.513^{* * *}$ & $0.436^{*}$ & 0.068 & 0.034 & 0.196 & 0.244 & 0.133 \\
\hline & $P$-value & 0.453 & 0.620 & 0.328 & 0.012 & 0.096 & 0.126 & 0.156 & 0.003 & 0.014 & 0.716 & 0.854 & 0.290 & 0.185 & 0.477 \\
\hline \multirow[t]{3}{*}{ pref } & $\mathrm{CC}$ & 0.139 & -0.016 & -0.041 & 0.037 & 0.058 & -0.109 & -0.071 & 0.040 & 0.103 & -0.318 & 0.338 & 0.066 & -0.188 & -0.020 \\
\hline & $P$-value & 0.455 & 0.934 & 0.827 & 0.842 & 0.757 & 0.558 & 0.705 & 0.831 & 0.580 & 0.082 & 0.063 & 0.723 & 0.311 & 0.916 \\
\hline & $\mathrm{N}$ & 31 & 31 & 31 & 31 & 31 & 31 & 31 & 31 & 31 & 31 & 31 & 31 & 31 & 31 \\
\hline
\end{tabular}

ES, Equilibrium Score; K-BNT, Korean version of Boston Naming Test; R-CFT, Rey complex figure test; SVLT, Seoul Verbal learning Test; COWAT, Controlled Oral Word Association Test; SOT, Sensory organization test; CC, Correlation coefficient; SA, Sensory analysis; N, Number. a Spearman's correlation.* Correlation is significant at the 0.05 level (2-tailed). ** Correlation is significant at the 0.01 level (2-tailed). 
Supplementary Table 3: The correlation between motor latencies for each condition and each domain of neuropsychological test

\begin{tabular}{|c|c|c|c|c|c|c|c|c|c|c|c|c|c|c|c|}
\hline ML & & $\begin{array}{c}\text { Digit } \\
\text { span } \\
\text { forward }\end{array}$ & $\begin{array}{l}\text { Digit span } \\
\text { backward }\end{array}$ & $\begin{array}{c}\text { Naming } \\
\text { (K-BNT) }\end{array}$ & $\begin{array}{l}\text { R-CFT } \\
\text { copy }\end{array}$ & $\begin{array}{c}\text { SVLT } \\
\text { free recall }\end{array}$ & $\begin{array}{c}\text { SVLT } \\
\text { delayed } \\
\text { recall }\end{array}$ & $\begin{array}{l}\text { SVLT } \\
\text { recogni- } \\
\text { tion }\end{array}$ & $\begin{array}{l}\mathrm{R} \text {-CFT } \\
\text { immediate } \\
\text { recall }\end{array}$ & $\begin{array}{c}\mathrm{R}-\mathrm{CFT} \\
\text { delayed } \\
\text { recall }\end{array}$ & $\begin{array}{c}\mathrm{R}-\mathrm{CFT} \\
\text { recognition }\end{array}$ & $\begin{array}{l}\text { COWAT } \\
\text { Category } \\
\text { fluency }\end{array}$ & $\begin{array}{l}\text { COWAT } \\
\text { Letter } \\
\text { fluency }\end{array}$ & $\begin{array}{l}\text { Stroop word } \\
\text { reading }\end{array}$ & $\begin{array}{l}\text { Stroop color } \\
\text { reading }\end{array}$ \\
\hline \multirow[t]{2}{*}{ SBL } & $\mathrm{CC}$ & 0.097 & -0.021 & -0.106 & 0.038 & -0.238 & -0.074 & 0.027 & 0.028 & 0.022 & 0.255 & 0.009 & -0.089 & -0.060 & -0.112 \\
\hline & $P$-value & 0.603 & 0.911 & 0.571 & 0.840 & 0.197 & 0.694 & 0.884 & 0.880 & 0.905 & 0.165 & 0.963 & 0.636 & 0.747 & 0.548 \\
\hline \multirow[t]{2}{*}{ SFL } & $\mathrm{CC}$ & -0.050 & -0.035 & -0.129 & -0.045 & -0.156 & 0.057 & -0.036 & 0.055 & 0.036 & 0.177 & -0.255 & -0.258 & -0.027 & -0.145 \\
\hline & $P$-value & 0.789 & 0.853 & 0.488 & 0.812 & 0.403 & 0.759 & 0.848 & 0.768 & 0.849 & 0.341 & 0.167 & 0.161 & 0.885 & 0.435 \\
\hline \multirow[t]{2}{*}{ MBL } & $\mathrm{CC}$ & 0.103 & -0.123 & -0.086 & 0.079 & -0.190 & -0.276 & -0.219 & 0.029 & -0.035 & 0.016 & -0.097 & -0.155 & -0.019 & 0.041 \\
\hline & $P$-value & 0.582 & 0.511 & 0.645 & 0.672 & 0.305 & 0.133 & 0.236 & 0.876 & 0.853 & 0.930 & 0.604 & 0.405 & 0.918 & 0.827 \\
\hline \multirow[t]{2}{*}{ MFL } & $\mathrm{CC}$ & 0.106 & -0.162 & -0.017 & 0.005 & -0.076 & 0.028 & -0.031 & 0.082 & 0.117 & 0.174 & 0.085 & 0.155 & 0.057 & 0.021 \\
\hline & $P$-value & 0.570 & 0.383 & 0.926 & 0.979 & 0.683 & 0.881 & 0.868 & 0.661 & 0.532 & 0.348 & 0.650 & 0.404 & 0.759 & 0.910 \\
\hline \multirow[t]{2}{*}{ LBL } & CC & -0.084 & $-0.364 *$ & -0.303 & -0.188 & $-0.440^{*}$ & -0.324 & -0.246 & -0.160 & -0.078 & 0.018 & -0.307 & $-0.537 * *$ & -0.141 & $-.389(*)$ \\
\hline & $P$-value & 0.654 & 0.044 & 0.098 & 0.311 & 0.013 & 0.075 & 0.183 & 0.389 & 0.678 & 0.926 & 0.093 & 0.002 & 0.449 & 0.030 \\
\hline \multirow[t]{3}{*}{ LFL } & CC & -0.175 & -0.208 & -0.283 & -0.203 & -0.119 & 0.107 & -0.052 & -0.048 & 0.016 & -.024 & -0.126 & -0.245 & 0.004 & -0.202 \\
\hline & $P$-value & 0.347 & 0.262 & 0.122 & 0.273 & 0.523 & 0.568 & 0.781 & 0.798 & 0.931 & 0.899 & 0.499 & 0.184 & 0.982 & 0.275 \\
\hline & $\mathrm{N}$ & 31 & 31 & 31 & 31 & 31 & 31 & 31 & 31 & 31 & 31 & 31 & 31 & 31 & 31 \\
\hline
\end{tabular}

ES, Equilibrium Score; K-BNT, Korean version of Boston Naming Test; R-CFT, Rey complex figure test; SVLT, Seoul Verbal learning Test; COWAT, Controlled Oral Word Association Test; ML, motor latency; SBL, small backward latency; SFL, small forward latency; MBL, medium backward latency; MFL, medium forward latency; LBL, large backward latency; LFL, large forward latency;

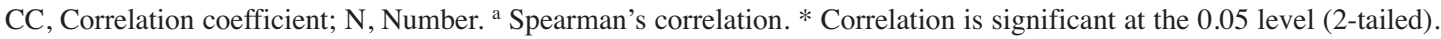

** Correlation is significant at the 0.01 level (2-tailed)

Supplementary Table 4: Comparison of median ES between this present study and previously reported study

\begin{tabular}{ccc}
\hline & $\begin{array}{c}\text { Untreated early stage PD } \\
\text { (Present study) } \\
\mathrm{N}=30\end{array}$ & $\begin{array}{c}\text { Untreated early stage } \\
\text { PD (Frenklach A study) } \\
\text { N=18 }\end{array}$ \\
\hline SOT condition(\%) & & \\
SOT 1 & 93.00 & 95.33 \\
SOT 2 & 89.00 & 91.83 \\
SOT 3 & 89.00 & 91.17 \\
SOT 4 & 74.00 & 85.00 \\
SOT 5 & 54.75 & 68.67 \\
SOT 6 & 54.42 & 68.50 \\
\hline
\end{tabular}

SOT, Sensory organization test; PD, Parkinson's disease; H-Y stage, Hoehn-Yahr stage.

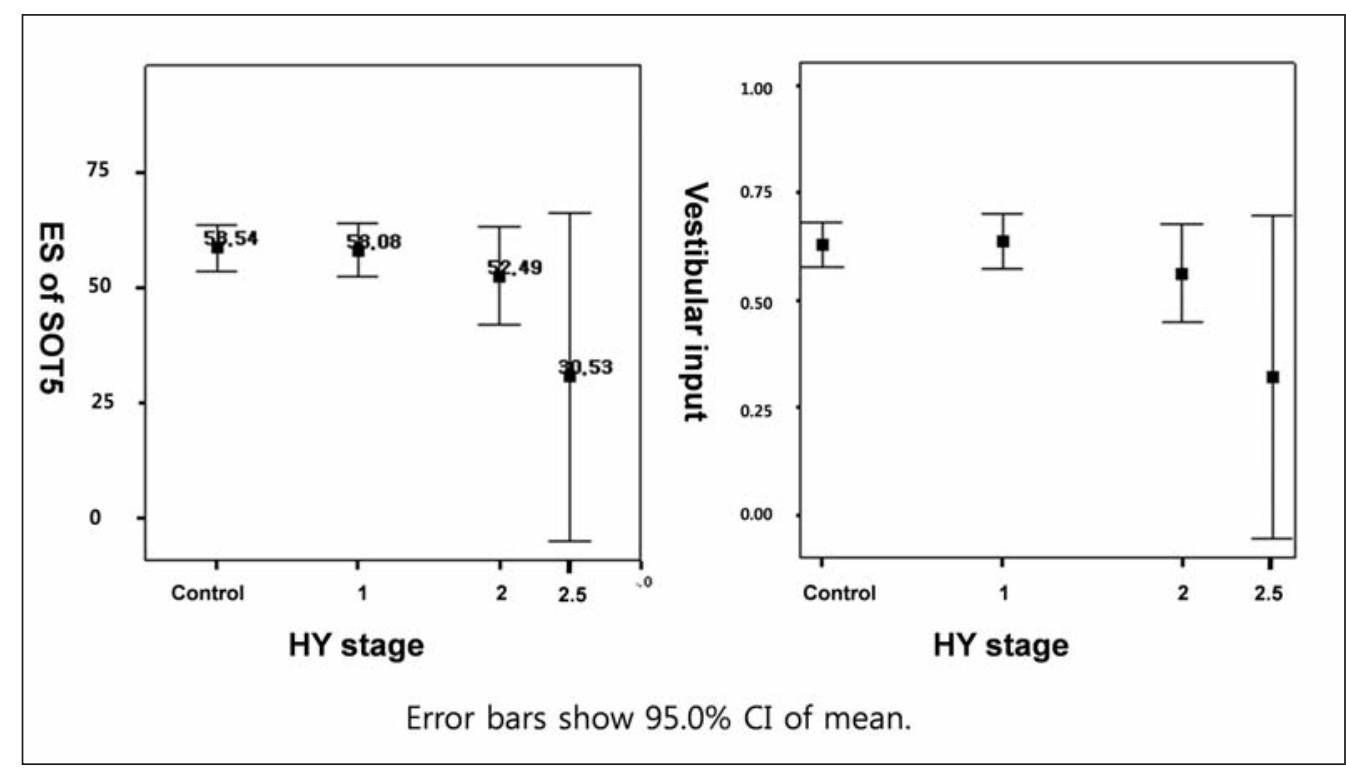

Supplementary Figure 1: The relationship between $H-Y$ stage and ES for SOT 5, and between H-Y stage and Vestibular dysfunction ratio ( $p<0.05)$. H-Y stage, Hoehn-Yahr stage; ES, Equilibrium score. 


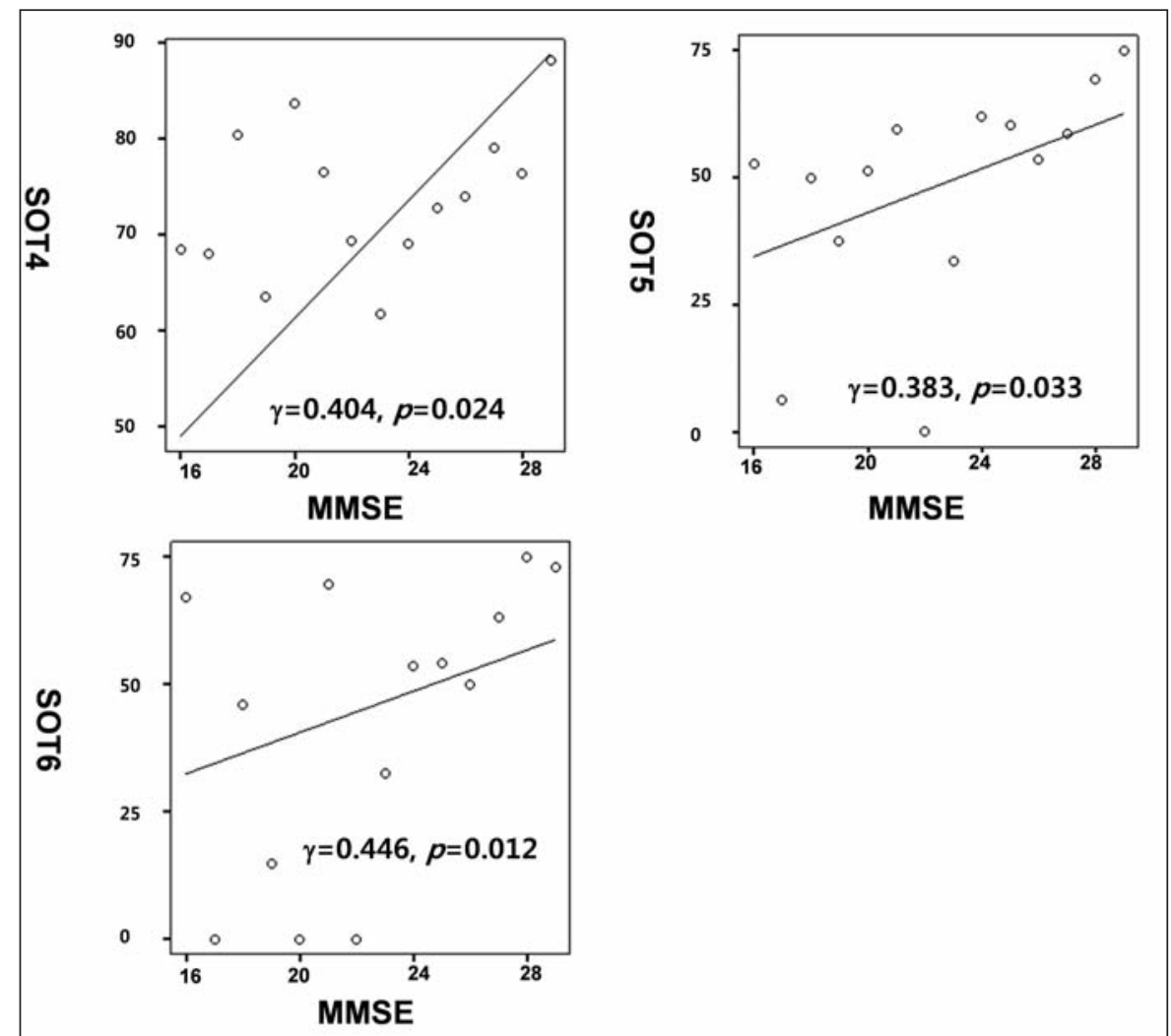

Supplementary Figure 2: The correlation between MMSE score and mean ES for SOT4, 5, and 6 conditons. ES, Equilibrium score; SOT, Sensory organization test; MMSE, Mini-mental state examination. 